British Journal of Industrial Medicine 1985;42:721-722

\title{
Editorial
}

\section{Radon daughter exposure and lung cancer}

What Agricola and Paracelsus described in the sixteenth century as "pulmonary consumption" among miners were probably the first case reports on lung cancer due to exposure to radon, an exposure that, today, is a matter of concern not only to miners but also in some countries to the general population.

Radon, discovered by Rutherford in 1899 , is a noble gas created by the decay of radium-226 in the uranium decay chain. When radon decays in the air, its short lived radioactive daughters (isotopes of polonium, bismuth, and lead) tend to attach to surfaces and to aerosol particles such as dust and cigarette smoke. Hence, through inhalation of radon daughters (and to a minor degree radon) the epithelium is radiated. Although radon daughters give off alpha, beta, and gamma radiation, it is the alpha radiation that delivers the significant dose to the tracheal and bronchial epithelium. The basal stem cells of the respiratory epithelium are considered to be those predominantly at risk after the inhalation of radon daughters.

The acute toxicity of radon was established in the early twentieth century and later studies showed that exposure to radon could induce lung tumours, both benign and malignant, and other cancers in experimental animals. A dose response relationship was established showing that the efficiency of radon and radon daughter exposure increased as cumulative exposure decreased.

Radium in the ground is the major source of radon outdoors but other sources contribute both outdoors and indoors-for example, natural gas, building materials, and ground water, especially from deep wells. The concentration of radioactivity in the ground varies considerably and the emanation of radon may show great differences within the same area. Radon is carried into the mines, particularly through deep underground water streams, but will also diffuse out of the rocks and from crushed ore; the miner gets exposed from breathing the mine atmosphere. Exposure to the general population occurs from indoor radon concentrations built up from the leakage of radon into houses from the geological materials in the ground and walls. The concentrations of radon and its daughters are heavily influenced by ventilation and by the presence of aerosols.

In 1879 Herting and Hesse reported the neoplastic nature of the lung disease among the miners in Schneeberg in Germany. Later, similar findings were reported from Joachimsthal in Czechoslovakia. For several decades the high mortality from lung cancer among these miners was thought of as a rare example of radiation induced occupational lung cancer. Today, however, several groups of miners have been shown to have a high incidence of lung cancer and with a few exceptions, coal mining and potash mining, for example, it seems as if underground mining involves an appreciable excess risk of lung cancer due to exposure to radon and its daughters. In various epidemiological studies the relative risk estimates are between four and 21 , suggesting that about $70-90 \%$ of the lung cancers among underground miners might be due to occupational exposure to radon and its daughters. Although the environment in the mines may have a modifying effect on the lung cancer rate, there is an acceptable agreement in the risk estimates, when different mining populations are compared, the excess of lung cancer being closely related to the cumulative radiation dose.

In the early studies of United States uranium miners almost every miner with lung cancer was a smoker and there was clearly a multiplicative interaction between smoking and radon daughter exposure. Consequently, smoking was thought to be the major contributor to the risk of lung cancer. This pattern remained in a follow up of these miners until December $1977,{ }^{1}$ suggsting that only a small fraction of lung cancer in non-smokers was due to radon daughter exposure. Other studies, however, showed an additive effect between smoking and radon daughter exposure. ${ }^{2}$ In some studies the average latent period from start of mining until death from lung cancer was about 3-12 years shorter in smokers than in nonsmokers. This could indicate that the major effects of tobacco smoke are exerted in the later phases of cancer development and may be those of a promoter, radiation acting in the early stages as an initiator. There is experimental work supporting this view, which also gains support from the experience that the risk of lung cancer in ex-smokers, 10-15 years after cessation of smoking, is only slightly greater than that of non-smokers. This would lead to an expectation of a multiplicative effect between radon daughter exposure and smoking, as also seen in some studies of miners. Under certain circumstances, however, smoking may influence mucous secretion and cause hyperplasia of the epithelium in the respiratory tract, 
thereby reducing the ability of the short range alpha particles effectively to penetrate to the basal cells of the epithelium and induce carcinogenic change. There is support for such a mechanism in an animal experiment in which smoking dogs developed fewer respiratory cancers than non-smoking dogs when exposed to radon daughters and uranium ore dust. ${ }^{3}$ Smoking miners are also known to have a relatively higher prevalence of bronchitis, which might perhaps explain why some studies have shown merely an additive effect between radon daughter exposure and smoking, for although the multiplicative effect might still obtain, the effective initiating dose may be reduced because of the increased mucous secretion in the respiratory tract, especially in populations from old dusty mines.

It has been suggested that the carcinogenic effect of radon daughter exposure may be restricted to specific histological types such as the small cell undifferentiated forms. This assumption seems to have been based on the early reports of the distribution of hitological types of bronchial cancer among uranium miners, indicating that the prevalence of oat cell and other small cell undifferentiated carcinomas (SCU) increased with increasing exposure to radiation in uranium mines. The appearance of other cancer types among uranium miners has now been reported with an increased incidence particularly of epidermoid cancers and adenocarcinomas. ${ }^{4}$ All three cancer types, SCU, epidermoid carcinoma, and adenocarcinoma, seem generally to be related to smoking. For miners at least the distribution of the various histological types of lung cancer may depend on a complex relation between smoking, aging, and latent period.

In many countries the inhalation of radon and radon daughters from indoor air constitutes a considerable part of the natural radiation dose. Low ventilation rates in modern homes tend further to build up the concentrations of radon and its daughters, and in view of the risk of lung cancer associated with the exposure among miners the presence of radon daughters in houses has become a matter of concern in several countries. In Sweden measurements made by various local and regional health authorities have indicated that the radioactivity in the ground is most important for the leakage of radium into the houses. Concentrations of radon and its daughters in houses may be surprisingly high, and in about 35000 houses in Sweden the dose exceeds what is permitted in mines on the basis of a $\mathbf{4 0}$ hour week working time. Therefore, a lung cancer hazard is likely to be present and some epidemiological studies have suggested a relation between the concentration of radon daughter exposure in dwellings and lung cancer and a multiplicative effect with smoking. ${ }^{5}$

The quantification of adverse health effects is usually difficult and several reports have been compiled by national and international organisations in order to summarise the information obtained from different mining populations. Depending on the approach taken and the data selected a range of estimates may be extracted. This variation probably reflects in large part differences in the follow up time of the various populations and the lack of adequately age standardised risk estimates when comparing different mining groups. Many studies now indicate a risk of about 31.7 excess cases per $\mathrm{MBqh} / \mathrm{m}^{3}$ and 10 person-years (20 excess cases/WLM/10 $10^{6}$ PY) ${ }^{6}$ This estimate is at least four to six times greater than it was estimated to be 18 years ago and suggests that a risk of lung cancer extends to and below the current standard in many countries of $1100 \mathrm{~Bq} / \mathrm{m}^{3}(0 \cdot 3 \mathrm{WL})$. Some theoretical estimates of the magnitude of the public health hazards have been made indicating that about $10-40 \%$ of the incidence of lung cancer might be due to exposure to radon and its daughters. These calculations have usually been based on the risk estimates from various mining populations and an assumption of a linear relation between dose and effect. Others state that the low dose effects have been overestimated and that the attributable risk of lung cancer from exposure to radon daughters in the general population is considerably lower, about $1-5 \%{ }^{7}$

Recently some provocative hypotheses have been raised regarding the role for radon in causing lung cancer $^{8}$ and obviously further epidemiological studies are needed to elucidate more definitely the question of lung cancer and radon daughter exposure in the gen eral population and the interaction between radon daughter exposure and smoking.

Department of Occupational Medicine,

C EDLINC

University Hospital,

S-75185 Uppsala, Sweden:

\section{References}

${ }^{1}$ Whittemore AS, McMillan A. Lung cancer mortality among US uranium miners: a reappraisal. JNCI 1983;71:489-99.

${ }^{2}$ Axelson $\mathrm{O}$, Sundell L. Mining, lung cance and smoking. Scand $J$ Work Environ Health 1978;49:46-52.

${ }^{3}$ Cross FT, Palmer RF, Filipy RE, Dagle GE, Stuart BO. Carcinogenic effects of radon daughters, uranium ore dust and cigarette smoke in beagle dogs. Health Phys 1982;42:33-52.

${ }^{4}$ Saccomanno G, Archer VE, Auerbach O, et al. Age factor in histological type of lung cancer among uranium miners. Preliminary report. International conference on radiation hazards in mining, Golden Colorado, 1981. New York: Society of Mining Engineers of American Institute of Mining, Metallurgical and Petroleum Engineers Inc, 1981.

${ }^{5}$ Edling C, Kling H, Axelson O. Radon in homes-a possible cause of lung cancer. Scand J Work Environ Health 1984;10:25-34.

${ }^{6}$ Beir III. Committee on the biological effects of ionizing radiations: the effects on populations of exposure to low levels of ionizing radiation. Washington: National Academy of Sciences, 1980.

${ }^{7}$ Cohen BL. Failures and critique of the BEIR III lung cancer risk estimates. Health Phys 1982;42:267-84.

${ }^{8}$ Axelson $O$. Room for a role for radon in lung cancer causation? Med Hypotheses 1984;13:51-61. 